\title{
Monitoring of Patients with Rheumatoid Arthritis by Indocyanine Green (ICG) enhanced Fluorescence Optical Imaging Treated with anti-TNFa Therapy
}

Simon Hertrampf

Charité - Universitätsmedizin Berlin

Jens Klotsche

Deutsches Rheumaforschungszentrum (DRFZ) Berlin, Leibniz Research Network

Quirino Schefer

regenold $\mathrm{GmbH}$

Anne-Marie Glimm

University Hospital Leipzig

Gerd-Rüdiger Burmester

Charité - Universitätsmedizin Berlin

Paula Hoff

Endokrinologikum Berlin am Gendarmenmarkt

Gabriela Schmittat

Charité - Universitätsmedizin Berlin

Thomas Häupl

Charité - Universitätsmedizin Berlin

Sandra Hermann

Charité - Universitätsmedizin Berlin

Marina Backhaus

Park-Klinik Weißensee, Berlin

Sarah Ohrndorf ( $\nabla$ sarah.ohrndorf@charite.de)

Charité - Universitätsmedizin Berlin

\section{Research Article}

Keywords: Fluorescence Optical Imaging, anti-TNFa therapy, certolizumab, rheumatoid arthritis, inflammation

Posted Date: February 25th, 2022

DOI: https://doi.org/10.21203/rs.3.rs-1369494/v1 
License: (c) (i) This work is licensed under a Creative Commons Attribution 4.0 International License. Read Full License 


\section{Abstract}

\section{Background:}

Fluorescence Optical Imaging (FOI) enables visualization of inflammation in both hands in rheumatoid arthritis (RA).

\section{Objective:}

To investigate FOI in treatment monitoring under anti-TNFa therapy with Certolizumab pegol (CZP) in patients with RA in comparison to clinical and laboratory outcome parameters.

\section{Methods:}

CZP-naïve patients with RA were eligible for this open-label study with an observational period of 52week. Disease activity was monitored by the clinical score DAS28, tender/swollen joint count (TJC28/SJC-28) and laboratory outcomes for systemic inflammation (CRP and ESR). FOI results were analyzed in three different phases (P1-3) and PrimaVistaMode (PVM) by the FOI activity score (FOIAS).

\section{Results:}

Twenty-eight RA patients (median age 52.5 years, 26 females, thirteen with a history of other biologic therapy) were included. DAS28(CRP) decreased from moderate disease activity at baseline (median 4.6, IQR 1.8) to low disease activity at week (w) 52 (median 2.7, IQR $2.1 ; \mathrm{p}<0.001)$. Statistically significant decreases could also be demonstrated for SJC-28 and TJC-28. CRP/ESR were reduced numerically from baseline to w52. FOIAS in P1 (early phase) showed a continuous decrease of enhancement during the course of treatment period: from baseline (median 1.5, IQR 9.3) over w6 (median 1.0, IQR 3.0; $p=0.069$ ), w12 (median 0.5, IQR 3.0; $p=0.171)$, w24 ( $n=27$, median 0.0, IQR 3.0; $p=0.004)$, until w52 ( $=18$, median 0.0, IQR 2.8; $p=0.091$ ), which could not be presented for FOIAS in P2, P3 and PVM.

\section{Conclusion:}

FOI in P1 appears to be a valuable tool for fast and easy monitoring of treatment response to certolizumab in a clinical setting.

\section{Introduction}

Rheumatic and musculoskeletal diseases (RMDs) represent a heterogeneous group with a high prevalence of around $22 \%$ in adults in the United States (US)(1). Even though there is a wide range of novel therapeutics, inflammatory arthritis still causes reduced mobility, limited quality of life and high health care costs $(1,2)$. Among RMDs, rheumatoid arthritis (RA) is the most common one with a prevalence of $0.5-1.0 \%(3,4)$. In order to reduce the effect of chronic joint inflammation, present therapeutic strategy builds on early recognition during the "window of opportunity" for an effective therapy improving the short and long term outcome significantly $(5,6)$. 
Inhibitors of tumor necrosis factor alpha (TNFa) were the first developed among biological medications and they dramatically changed the therapeutic perspectives of RA patients(7). Follow-up studies investigating their clinical and radiographic effects have shown that TNFa inhibitors, especially in combination with conventional synthetical disease-modifying antirheumatic drugs (csDMARD), lead to significant improvements in clinical status and significant inhibition of radiographic progression.

In order to monitor the therapeutic response in RA, several options are applied, such as different clinical disease activity scores, e.g., DAS28(8). For early and more objective monitoring of RA disease activity, musculoskeletal ultrasound (MSUS) and magnetic resonance imaging (MRI) are used in clinical practice(9-12). In several clinical studies, MSUS presented sensitivity in disease changes under therapy, especially in power Doppler mode (PD)-detected synovitis and tenosynovitis $(13,14)$. Different joint combinations have been used for MSUS scoring. The US7-joint score firstly proposed by Backhaus et al. has been the most largely utilized score in the literature and includes five hand and two foot joints of the clinically dominant side(15).

More recently, after its first report in 2006(13) and the launch of a commercial device in 2009 (Xiralite $\mathrm{GmbH}$, Berlin; Germany), fluorescence optical imaging (FOI) has been shown to detect inflammation in preclinical studies as well as in humans in the joint regions of both hands(16-21). Basis of the Xiralite method is the illustration of an impaired microcirculation caused by the inflammatory process of arthritis. For that, the enhancement of the intravenously applied dye indocyanine green (ICG) is evaluated. Accordingly, FOI is a non-ionizing technique that examines both hands in one session of six minutes.

The aim of this study was to investigate FOl's ability to monitor the treatment response to TNFa inhibitor therapy with certolizumab pegol (CZP) in RA patients and to compare FOl findings to clinical outcome parameters such as DAS28(CRP), tender joint count (TJC-28), swollen joint count (SJC-28), and laboratory parameters. The US7 score was set as further imaging method with high sensitivity to inflammation.

\section{Patients And Methods}

Patients with RA according to 2010 ACR/EULAR classification criteria(22) have been enrolled after written informed consent to participate in the study. They were examined at baseline (before CZP) and after 6,12 , 24 and 52 weeks. Eligible for participation in the study were adults (age of $\geq 18$ years), CZP-naïve (not naïve for other biologic therapies) patients with active RA (DAS28(CRP)) with tolerability of the drug's ingredients and no known heart failure (NYHA III/IV).

\section{Clinical and laboratory examination}

During all visits, the disease activity score $28(\operatorname{DAS} 28)(23,24)$ including clinical assessments of tender (TJC-28), swollen joints (SJC-28) and patient's self-reported global disease activity on a Visual Analogue Scale (VAS) from 0 to $10 \mathrm{~cm}$ was assessed. Furthermore, physician's global assessment (PGA) and morning stiffness (in minutes) were evaluated. The laboratory investigation included the assessment of erythrocyte sedimentation rate (ESR; normal value $<20 \mathrm{~mm} / \mathrm{h})$ and C-reactive protein (CRP; normal value < 
$5.0 \mathrm{mg} / \mathrm{l})$. Clinical and laboratory examination was accomplished on the same day as the imaging examinations (FOl; US7 score).

\section{Fluorescence optical imaging (FOI)}

The FOI system (Xiralite) was used following a standardised procedure with an examination term of six minutes, recording one image per second, and resulting in a cluster of 360 images. A bolus of indocyanine green (ICG) as fluorescence optical dye with a dose of $0.1 \mathrm{mg} / \mathrm{kg}$ body weight was injected intravenously ten seconds after beginning the examination $(20,21)$. To evaluate the distribution of ICG, the image sequence in the film modus was evaluated on one hand. For this purpose, three phases in position to the fingertips were defined regarding development of signal intensities that depend on the dye flooding of the individual patient(21). Phase 1 (P1) includes the period between starting the examination, application of the dye and increased signal intensities in the fingertips(21). When the dye leaves the fingertips from distal to proximal, phase 2 (P2) begins as the period of persisting high signal intensities in the fingertips(21). It can be identified by the red colour in the fingertips. Phase 3 (P3) starts when no signal intensity can be determined in the fingertips, i.e. the time point without signal intensity (only yellow sparkles) in the fingertips as signal for clearance(21). In addition, an electronically generated composite image (Prima Vista Mode, PVM) automatically obtained by means of 240 images with the integrated software XiraView was analysed.

For analysing the joint activity by FOI, 'FOIAS' (Fluorescence optical imaging activity score) as a semiquantitative grading system was applied, based on individual joint scores from $0-3$. The scores in P1-3 and in PrimaVistaMode (PVM) were assessed for 30 joints per patient, including the bilateral wrist, metacarpophalangeal joints (MCP) I-V, proximal interphalangeal joints (PIP) II-V, distal interphalangeal joints (DIP) II-V and interphalangeal joint of the thumb (IP). In addition, the sum scores of all affected joints of the left hand and the right hand were individually calculated and represented as one sum score (sum score both hands)(25). FOIAS was performed by an agreement-based consensus of two investigators (SO, SH).

\section{Musculoskeletal ultrasound (MSUS)}

Ultrasonographic examination was performed in greyscale (GS) and power Doppler (PD) mode with a linear 10-18 MHz transducer (Esaote Mylab Twice,Genova; Italy). The examined level of the wrist included the dorsomedian, palmomedian and ulnar plane. The metacarpophalangeal (MCP) 2,3, the proximal interphalangeal (PIP) 2,3 and the metatarsophalangeal (MTP) 2,5 joints were examined from the palmar/plantar and dorsal view in the longitudinal plane. Tenosynovitis was measured on longitudinal and transverse scans only at the wrist and MCP 2,3 joint level. The wrist, MCP/PIP2,3 and MTP 2,5 joints were examined for synovial effusion and/or proliferation (indicating synovitis) according to Scheel et al. (26) in GSUS and according to Szkudlarek et al.(27) in PDUS (each 0-3), and for signs of tenosynovitis in GS (0-1)/PDUS (0-3)(15). The US7 score was used in a modified way also including MCP2,3 and PIP2,3 from the dorsal view in GS(28). The scoring range was $0-39$ for GS and PD synovitis, $0-5$ for GS tenosynovitis (0-5), and $0-15$ for PD tenosynovitis 


\section{Statistical analyses}

A two-sided Wilcoxon signed rank test was used to explore the difference in central tendency of the clinical data, FOI and MSUS scores (each 0-3, except tenosynovitis in GS US7 score) between baseline and follow-up visits. In addition, we examined whether the FOI was correlated with the clinical outcome using Spearman correlation, since a considerable number of tied values was to be expected. Furthermore, the resulting correlation coefficients were tested for their difference from zero, i.e., no correlation. Statistical analyses were performed with the statistical program SPSS. If not specified otherwise, the descriptive statistics provided median values (min-max; interquartile range [IQR]). For FOI scores, the sum of all joints and both hands were evaluated (sumscore both hands). For US7 scores, the sum of all subscores were evaluated. Whenever meaningful, p-values were provided based on the underlying methods. These explorative p-values were used to highlight statistical noticeable differences.

\section{Results}

In this study, 28 patients with RA were included and treated with $400 \mathrm{mg}$ CZP s.c. at weeks 0,2 and 4 and every 2 weeks thereafter with $200 \mathrm{mg}$ s.c.. Of these patients, all received CZP for at least 12 weeks, $n=27$ (96.4\%) until w24, and $n=18$ (64.3\%) received CZP throughout the entire 52 weeks. The reasons for dropout were withdrawal of CZP due to lack of efficacy $(n=9)$ or worsening of symptoms $(n=1)$. Fifteen patients were biologic naïve at inclusion. Thirteen patients had a history of biologic treatment. Sixteen patients were on background therapy with methotrexate who received a mean dosage of $16 \pm 4.28 \mathrm{mg}$ per week and 23 patients with prednisolone. During the course of the study, the mean dosage of prednisolone decreased from $6.0 \pm 4.75 \mathrm{mg}$ per day to $3.9 \pm 2.60 \mathrm{mg} / \mathrm{d}$ at $\mathrm{w} 24$ and $2.3 \pm 2.26 \mathrm{mg} / \mathrm{d}$ at $w 52$.

Patients' characteristics at baseline are presented in Table 1. 
Table 1

Baseline Characteristics

\begin{tabular}{|c|c|c|}
\hline & $\mathbf{N}$ & \\
\hline Age (years) & 28 & $52.5(22-75 ; 20.0)$ \\
\hline Sex (female), n (\%) & 28 & $26(92.9)$ \\
\hline Weight (kg) & 28 & $75.5(50-105 ; 24.3)$ \\
\hline Disease duration (months) & 28 & $31.5(4-180)$ \\
\hline \multicolumn{3}{|l|}{ Medication } \\
\hline Methotrexate, n (\%) & 28 & $16(57.1)$ \\
\hline Prednisolone, n (\%) & 28 & $23(82.1)$ \\
\hline Biologics naïve, n (\%) & $25^{\star}$ & $15(60.0)$ \\
\hline \multicolumn{3}{|l|}{ Clinical parameters } \\
\hline DAS28(CRP) & 28 & $4.61(2.65-6.49 ; 1.75)$ \\
\hline Tender joint count (28-TJC) & 28 & $7.0(0-22 ; 8.75)$ \\
\hline Swollen joint count (28-SJC) & 28 & $2.5(0-9 ; 4.0)$ \\
\hline VAS Patient $(0-10 \mathrm{~cm})$ & 28 & $6.0(2.4-10 ; 3.0)$ \\
\hline VAS Physician $(0-10 \mathrm{~cm})$ & 28 & $4.0(1.9-8.5 ; 1.7)$ \\
\hline Morning stiffness (min.) & 28 & $30(0-240 ; 63.75)$ \\
\hline \multicolumn{3}{|l|}{ Laboratory parameters } \\
\hline $\mathrm{ESR}(\mathrm{mm} / \mathrm{h})$ & 28 & $27(8-66 ; 21.0)$ \\
\hline $\mathrm{CRP}(\mathrm{mg} / \mathrm{l})$ & $27^{\star \star}$ & $5.4(0.3-52 ; 17.4)$ \\
\hline \multicolumn{3}{|l|}{ Fluorescence optical imaging ${ }^{\dagger}$} \\
\hline P1 & 28 & $1.5(0-16 ; 9.25)$ \\
\hline $\mathrm{P} 2$ & 28 & $19.5(0-36 ; 14.5)$ \\
\hline P3 & 28 & $1.0(0-20 ; 3.75)$ \\
\hline PVM & 28 & $5(0-27 ; 6.25)$ \\
\hline \multicolumn{3}{|c|}{ Musculoskeletal ultrasound (US7 Score) } \\
\hline GS US7 Synovitis (range 0-39) & 28 & $11(4-22 ; 8.25)$ \\
\hline PD US7 Synovitis (range 0-39) & 28 & $2.0(0-17 ; 3.0)$ \\
\hline
\end{tabular}




\section{$\mathbf{N}$}

GS US7 Tenosynovitis (range 0-5)

PD US7 Tenosynovitis (range $0-15$ )
28

28
$0.5(0-3 ; 1.0)$

$0(0-2 ; 1.0)$

Data represent median (min-max;interquartile range) unless otherwise stated. *Status for 3 patients unknown; **one sample not analysed; ${ }^{*}$ sum scores of both hands. DAS28 $=$ disease activity score of 28 joints; $C R P$ = C-reactive protein; VAS = visual analogue scale; $E S R$ = erythrocyte sedimentation rate; GS = grey scale; PD = Power Doppler; P: phase, PVM: prima vista mode; US7: ultrasound score of 7 selected joints.

\section{Clinical and laboratory outcome parameters}

Disease activity measured by DAS28(CRP) continuously decreased from baseline up to w52. At baseline, patients had a moderate to high disease activity with a median (min-max; IQR) DAS28(CRP) of 4.6 (2.7$6.5 ; 1.8)$, which decreased under treatment to $3.2(1.3-6.4 ; 1.8 ; p=0.001)$ at w24 and to $2.7(1.2-4.9 ; 2.1$; $\mathrm{p}<0.001)$ at $w 52$.

Similar results were observed for SJC-28 and TJC-28, which are also part of the DAS28(CRP). Both outcome parameters continuously decreased from baseline up to w52. For SJC-28, a decrease from median of $2.5(0-9,4.0)$ at baseline to $0.0(0-6,2.0 ; p=0.005)$ at $w 24$, and to $0.0(0-6-1.8 ; p=0.012)$ at w52 was observed. For TJC-28, a decrease from median of $7.0(0-22,8.8)$ at baseline to $3.0(0-17,4.0 ; p$ $=0.001)$ at $w 24$ and to $2.0(0-15,3.5 ; p=0.004)$ at w52 was observed.

Patient Global Assessment (VAS) decreased during the course of the study. Significant differences were observed at w6, w12, and w52. Similar results were observed for the Global Assessment performed by the physician.

Morning stiffness decreases continuously from baseline to $w 52$ with significant findings at $w 6, w 12$, and w24.

CRP and ESR values continuously decreased from baseline to w52. The median CRP decreased from 5.4 $\mathrm{mg} / \mathrm{l}(0.3-52 ; 17.4)$ to $2.3 \mathrm{mg} / \mathrm{l}(0.3-41 ; 5.5 ; \mathrm{p}=0.028)$ at w24 and to $2.0 \mathrm{mg} / \mathrm{l}(0.2-99 ; 5.0 ; \mathrm{p}=0.156)$ at w52. The median ESR decreased from $27 \mathrm{~mm} / \mathrm{h}(8-66 ; 21)$ to $21 \mathrm{~mm} / \mathrm{h}(2-70 ; 25.5 ; \mathrm{p}=0.203)$ at w24 and to $18 \mathrm{~mm} / \mathrm{h}(2-54 ; 20 ; p=0.177)$ at w52.

A summary of all measured clinical and laboratory outcome parameters is listed in Table 2. 
Table 2

Summary statistics of clinical and laboratory outcome parameters at baseline and after w6, w12, w24 and w52

\begin{tabular}{|c|c|c|c|}
\hline Clinical Outcome Parameters & $\mathbf{N}$ & Median (min-max, IQR) & p-value \\
\hline \multicolumn{4}{|l|}{ DAS28(CRP) } \\
\hline baseline & 28 & $4.61(2.65-6.49,1.76)$ & \\
\hline w6 & 28 & $3.80(1.49-6.60,1.88)$ & 0.002 \\
\hline w12 & 27 & $3.26(1.39-6.65,2.04)$ & $<0.001$ \\
\hline w24 & 27 & $3.20(1.27-6.37,1.81)$ & 0.001 \\
\hline w52 & 18 & $2.73(1.22-4.85,2.06)$ & $<0.001$ \\
\hline \multicolumn{4}{|l|}{ Tender Joint Count-28 } \\
\hline baseline & 28 & $7.00(0-22,8.75)$ & \\
\hline w6 & 28 & $3.00(0-22,7.00)$ & 0.009 \\
\hline w12 & 28 & $4.00(0-21,5.00)$ & $<0.001$ \\
\hline w24 & 27 & $3.00(0-17,4.00)$ & 0.001 \\
\hline w52 & 18 & $2.00(0-15,3.50)$ & 0.004 \\
\hline \multicolumn{4}{|l|}{ Swollen Joint Count-28 } \\
\hline baseline & 28 & $2.50(0-9,4.00)$ & \\
\hline w6 & 28 & $2.00(0-6,2.00)$ & 0.052 \\
\hline w12 & 28 & $1.00((0-7,3.00)$ & 0.009 \\
\hline w24 & 27 & $0.00(0-6,2.00)$ & 0.005 \\
\hline w52 & 18 & $0.00(0-6,1.75)$ & 0.012 \\
\hline \multicolumn{4}{|l|}{$\mathrm{CRP}(\mathrm{mg} / \mathrm{l})$} \\
\hline baseline & 28 & $5.40(0.30-52.00,17.36)$ & \\
\hline w6 & 27 & $4.00(0.30-61.87,11.85)$ & 0.687 \\
\hline w12 & 26 & $3.25(0.30-28.90,6.95)$ & 0.091 \\
\hline w24 & 25 & $2.30(0.30-41.40,5.50)$ & 0.028 \\
\hline w52 & 18 & $2.00(0.20-98.70,5.02)$ & 0.156 \\
\hline
\end{tabular}

IQR = interquartile range, DAS28 = disease activity score of 28 joints, ESR = erythrocyte sedimentation rate, $\mathrm{CRP}=\mathrm{C}$-reactive protein, VAS = visual analogue scale; significance level of $p<0.05$ 


\begin{tabular}{|c|c|c|c|}
\hline Clinical Outcome Parameters & $\mathbf{N}$ & Median (min-max, IQR) & p-value \\
\hline baseline & 28 & $27(8-66,21,00)$ & \\
\hline w6 & 28 & $20(4-52,24,50$ & 0.027 \\
\hline w12 & 28 & $18(6-56,23.25)$ & 0.059 \\
\hline w24 & 27 & $21(2-70,25.50)$ & 0.203 \\
\hline w52 & 18 & $18(2-54,20.00)$ & 0.177 \\
\hline \multicolumn{4}{|l|}{ VAS $(0-10 \mathrm{~cm})$ patient } \\
\hline baseline & 28 & $6.00(2.40-10.00,3.00)$ & \\
\hline w6 & 28 & $2.90(0.00-8.50,3.95)$ & 0.003 \\
\hline w12 & 28 & $4.20(0.10-8.90,3.00)$ & 0.003 \\
\hline w24 & 27 & $5.00(0.30-9.00,3.70)$ & 0.112 \\
\hline w52 & 18 & $2.95(0-8.00,3.63)$ & 0.002 \\
\hline \multicolumn{4}{|l|}{ VAS $(0-10 \mathrm{~cm})$ physician } \\
\hline baseline & 28 & $4.00(1.9-8.50,1.70)$ & \\
\hline w6 & 28 & $3.00(1.0-7.50,2.00)$ & 0.002 \\
\hline w12 & 28 & $2.25(1.0-8.00,1.63)$ & 0.001 \\
\hline w24 & 27 & $2.00(0.5-10.00,2.25)$ & 0.066 \\
\hline w52 & 18 & $1.50(0.0-6.00,2.00)$ & 0.014 \\
\hline \multicolumn{4}{|l|}{ Morning stiffness (min.) } \\
\hline baseline & 28 & $30.00(0-240,63,75)$ & \\
\hline w6 & 28 & $2.50(0-120,30.00))$ & 0.003 \\
\hline w12 & 28 & $7.50(0-90,33.75)$ & 0.025 \\
\hline w24 & 27 & $0.00(0-120,45.00)$ & 0.042 \\
\hline w52 & 18 & $0.00(0-150,12.50)$ & 0.091 \\
\hline
\end{tabular}

\section{Fluorescence Optical Imaging (FOI)}

The sum score of both hands in P1 of FOI continuously decreased from baseline up to w52. A decrease in P1 of FOI sum score was already observed at w6, with a reduction from a median of $1.5(0-16,9.3)$ at 
baseline to $1.0(0-12,3 ; p=0.069)$ at w6. The FOI P1 1 sum score further decreased to $0.5(0-46,3 ; p=$ $0.171), 0.0(0-10,3 ; p=0.004), 0.0(0-13,2.8 ; p=0.091)$ at $w 12$, w24, and $w 52$, respectively.

A statistically significant reduction was detected at w24. Although the reduction from baseline remains at w52, the applied test did not yield a significant result. This occurred likely due to the reduced number of patients at that timepoint.

All other sum scores of both hands (PVM, P2 and P3) did not show a median reduction during the treatment.

The results of the FOI are presented in Table 3. 
Table 3

Summary statistics of FOI phases 1-3 and PMV at baseline, w6, w12, w24 and w52

\section{N Median (min-max, IQR)}

p-value

Phase 1

baseline

$28 \quad 1.5(0-16,9.25)$

w6

27

$1.0(0-12,3.00)$

0.069

w12

28

$0.5(0-46,3.00)$

0.171

w24

27

$0.0(0-10,3.00)$

0.004

w52

18

$0.0(0-13,1.75)$

0.091

Phase 2

baseline

28

$19.5(0-36,14.50)$

w6

27

$19.0(0-43,13.50)$

0.451

w12

28

$19.0(0-50,13.25)$

0.071

w24

27

$15.0(1-48,15.50)$

0.310

w52

18

$19.0(0-35.12-75)$

0.256

Phase 3

baseline

28

$1.0(0-20,3.75)$

w6

27

$2.0(0.22,3.50)$

0.501

w12

28

$2.5(0-32,6.25)$

0.089

w24

27

$1.0(0-17,3.00)$

0.191

w52

18

$0.5(0-12,2.00)$

0.959

PMV

baseline

28

$5.0(0-27,7.00)$

w6

27

$8.0(0-25,7.00)$

0.018

w12

28

$5.5(0-36,9.25)$

0.472

$\mathrm{FOI}=$ fluorescence optical imaging, $\mathrm{PMV}=$ PrimaVista mode, $\mathrm{IQR}$ = interquartile range; significance level of $p<0.05$

A) Baseline visit. FOI findings with clinical active RA. Increased signal intensities as a sign of active inflammation in P1. High signal intensities (FOIAS grade 3) in PIP5 of the right hand, moderate signal intensities (FOIAS grade 2) in both wrists and PIP4 of the right and PIP5 of the left hand.

B) After 52 weeks of CZP treatment; no increased signal intensities are detectable. 


\begin{tabular}{|llll|}
\hline & N & Median (min-max, IQR) & p-value \\
\hline w24 & 27 & $7.0(0-23,8.50)$ & 0.204 \\
\hline w52 & 18 & $5.5(0-23,5.75)$ & 0.717 \\
\hline
\end{tabular}

$\mathrm{FOI}=$ fluorescence optical imaging, $\mathrm{PMV}=$ PrimaVista mode, $\mathrm{IQR}$ = interquartile range; significance level of $p<0.05$

A) Baseline visit. FOI findings with clinical active RA. Increased signal intensities as a sign of active inflammation in P1. High signal intensities (FOIAS grade 3) in PIP5 of the right hand, moderate signal intensities (FOIAS grade 2) in both wrists and PIP4 of the right and PIP5 of the left hand.

B) After 52 weeks of CZP treatment; no increased signal intensities are detectable.

Figure 1 shows an example of a patient with good response to the CZP therapy which is displayed as a signal attenuation in comparison from baseline, visit 1 to week 52 in P1. The improvement of the inflammatory process can be seen in the significant decrease of early enhancement in both hands during the treatment period.

\section{Musculoskeletal ultrasound}

For GS, a numerical decrease of the US7 synovitis sum score was only observed at w52 (from 11.0 at baseline to 8.5 at w52).

For PD, a numerical decrease of the US7 synovitis score was detected at w12 (from 2.0 to 1.5) with further decrease at w52 (to 0.5).

For GS tenosynovitis und PD tenosynovitis US7 scores, no relevant changes could be detected throughout the study.

The outcome parameters of the GS and PD US7 synovitis/tenosynovitis scores are presented in the supplement Table S1 at baseline and after 6, 12, 24 and 52 weeks of therapy with CZP.

\section{Correlation of FOIAS with selected clinical and US7 Score outcome parameters}

Regarding baseline data, FOIAS in PMV and P1, P2, P3 showed no significant positive correlations with any clinical outcome parameter. There was a moderate correlation between FOIAS P3 with PD US7 synovitis score, and a weak correlation between FOIAS P1 and PD tenosynovitis score.

At week 24, FOIAS in PMV and P1, P2, P3 showed no significant positive correlations with any clinical outcome parameter. There was a weak correlation between FOIAS P3 with PD synovitis score.

At week 52, FOIAS P2 showed a moderate correlation with TJC-28. There was a moderate correlation between FOIAS P2, P3, PVM and PD synovitis score; and a moderate correlation between FOIAS P3, PVM 
and PD tenosynovitis score.

Pairwise correlations between clinical and laboratory outcomes with FOIAS P1-3 and PMV as well as with US7 score are presented in Figure S1 (Supplement).

Supplement Figure S2 presents box plots of DAS28, FOIAS P1, GS, PD, SJC and TJC at baseline, w6, w12, w24 and w52.

\section{Discussion}

In this open-label study, we are presenting for the first time that FOI assessments by means of short-term study visits allow to monitor treatment response early after initiation of anti-TNFa therapy. Already after a 6-week treatment period, FOI in P1 showed a reduction of ICG enhancement based on the FOIAS and at $w 24$, the reduction of the FOIAS compared to baseline was statistically significant $(p=0.004)$. However, at w52, no significant reduction of the FOIAS has been observed - most probably due to the low number of patients $(n=18)$ at $w 52$.

According to the results of this analysis, P1 seems to be the most useful FOI phase for monitoring treatment response.

These results are in line with previous results by Meier et al.(29) and Glimm et al.(25) presenting significant reductions in early signal intensities after 24 weeks and after one year of treatment, respectively. In addition, in the study of Glimm et al., also FOI P1 was the only FOI phase with significant changes over the treatment period of one year. In another study on osteoarthritis(17) as primary noninflammatory joint disease, OA patients exhibited less activity in FOI P 1 compared to joint inflammation in RA (though, high signal accumulation in P2). These results support the hypothesis that FOI P1 detects an acute inflammation.

Regarding clinical disease activity, DAS28(CRP) decreased significantly from a median of 4.6 to a median of 3.2 at w24. After 52 weeks of treatment, a low disease activity (median of 2.7) was observed. The good response of CZP treatment in RA patients could also be shown by a reduction of the prednisolone dosage, which decreased from $6.0 \pm 4.75 \mathrm{mg}$ per day to $2.3 \pm 2.26 \mathrm{mg} / \mathrm{d}$ at $\mathrm{w} 52$.

A correlation of DAS28(CRP) and P1 could not be observed, which again is in line with the findings by Glimm et al.(25). The lack of correlation may be caused by measuring different outcome parameters assessed through the clinical examination by DAS28 compared to the FOI method. Clinical disease activity is mainly assessed by swollen and tender joints and by patients' self-reported VAS. In contrast, FOI via the Xiralite method detects disturbed microcirculation in the joints of both hands.

Hypervascularization and neoangiogenesis of the synovial membrane are considered to be primary pathogenic mechanism for the development of rheumatoid pannus on the joint leading to bone destruction(30). 
Because of the subjective part of DAS28(CRP) in decision making during RA treatment leading to high variance for components of the DAS28(CRP), FOI represents a more objective method for therapy monitoring.

Another explanation for the lack of correlation between FOI results and clinical outcome parameters might be the positive findings in $\mathrm{FOI}$ in asymptomatic joints, indicating subclinical inflammation. Werner et al.(20) found that FOl showed positive findings in $45 \%$ of clinically asymptomatic joints.

In our study, we also included the US7 score for detection of synovitis and tenosynovitis. The US7 synovitis sum score decreased, but not initially after start of CZP therapy. For GS, a numerical decrease of the synovitis sum score was observed at w52 only and for PD, a decrease of the synovitis score was detected at w12 with further decrease at w52. Sarzi-Puttini et al.(31) found an improvement in PD ultrasound already after w8 of CZP therapy, which maintained to w52. Overall, the MSUS results are in line with other studies. In contrast to the fast response in PD scoring, which identifies active inflammation, the GS usually identifies late changes such as the morphology of synovial proliferation, which explains the late response in our study $(15,32-34)$. In spite of the positive results in clinical studies, the lack of operator-dependence and time-consuming procedures are limitations of MSUS(33).

Advantage of $\mathrm{FOI}$ is the fast imaging in a comfortable sitting position by using a machine which can be installed in every clinical setting. It is also a delegable tool performed by a medical assistance with a physician in the background. FOI be used in diagnostic and therapy monitoring setting. Here, therapy monitoring by means of short-term study visits is likely the most important role because biological therapies such as anti-TNFa therapies are highly effective, but expensive. Thus, to keep costs as low as possible and to provide the patient with the optimal treatment, it is important to know early and objective evidence of a response or non-response.

The use of the chosen imaging method depends on the treating physician's preference and the indication for imaging. Information on bone erosions will likely remain the domain of MRI, MSUS and X-ray. However, determination of the degree and extent of joint inflammation as well as for the diagnostic of subclinical inflammation are indications for using the FOI method. Because of the detection of early synovitis in symptomatic and asymptomatic patients, FOI might also be a tool in early clinical studies to detect treatment response.

A limitation of the current study is the open design and the small sample size. Treatment responders could also be influenced by other factors that were not considered into the analysis, e.g., wounds located over the joint. Another limitation of the FOI device is that the system can only image the hands, so far. Furthermore, the intravenous ICG administration before initiating the FOI procedure might limit the routine use in clinical practice.

\section{Conclusion}


To our knowledge, this is the first study showing that FOI can be used to monitor treatment response early after initiating anti-TNFa therapy. Significant reductions in FOIAS were found in P1 which appears to be the most relevant phase for monitoring treatment response.

In contrast to clinical outcome parameters, FOI represents - in our opinion - a more objective method for therapy-monitoring and may provide an additional tool to assess therapy response early after initiating therapy with biologics. It may also give the opportunity for an additional evaluation of inflammation of wrist and finger joints of RA patients in clinical studies. Consequently, decrease in disease activity could easily be monitored with the Xiralite system by quantifying the fluorescence signals in the early phase (= Phase 1) of signal acquisition.

Fluorescence optical imaging with the Xiralite system is an interesting alternative to other imaging methods, not only in terms of a fast and easy assessment, but also its pictorial output, making it easier to follow and understand for the patient, enabling higher acceptance of treatment measures.

\section{Abbreviations}

ACR American College of Rheumatology

ACPA Anti-cyclic citrullinated peptides

bDMARD Biological disease modifying anti-rheumatic drug

CRP C-reactive protein

csDMARD Conventional synthetic disease modifying anti-rheumatic drug

CZP Certolizumab pegol

DAS28 Disease activity score of 28 joints

ESR Erythrocyte sedimentation rate

EULAR European alliance of associations for rheumatology

FOI Fluorescence optical imaging

FOIAS Fluorescence optical imaging activity score

GS Greyscale (ultrasound)

ICG Indocyanine green

IgM-RF Immunoglobulin M-rheumatoid factor

IP Interphalangeal joint of the thumb 


$\begin{array}{ll}\text { IQR } & \text { Interquartile range } \\ \text { MCP } & \text { Metacarpophalangeal (joint) } \\ \text { MRI } & \text { Magnetic resonance imaging } \\ \text { MSUS } & \text { Musculoskeletal ultrasound } \\ \text { MTP } & \text { Metatarsophalangeal (joint) } \\ \text { NSAIDs } & \text { Non-steroidal anti-inflammatory drugs } \\ \text { NYHA } & \text { New York Heart Association } \\ \text { OMERACT } & \text { Outcome measures in rheumatology clinical trial } \\ \text { P } & \text { Phase } \\ \text { PGA } & \text { Physician's Global Assessment } \\ \text { PIP } & \text { Proximal interphalangeal (joint) } \\ \text { PD } & \text { Power Doppler (ultrasound) } \\ \text { PVM } & \text { PrimaVistaMode } \\ \text { RA } & \text { Rheumatoid arthritis } \\ \text { RMD } & \text { Rheumatic and musculoskeletal diseases } \\ \text { SJC-28 } & \text { Swollen joint count-28 } \\ \text { TNFa } & \text { Tumor necrosis factor a } \\ \text { TJC-28 } & \text { Tender joint count-28 } \\ \text { VAS Score } & \text { Ultrasound Seven Score } \\ \text { Visual analogue scale } \\ \text { M }\end{array}$

\section{Declarations}

Ethics approval and consent to participate: The study was approved by the local Medical Ethical Committee of the Charité - Universitätsmedizin, Berlin, Germany (. All patients provided informed consent to participate in the study.

Consent for publication: All patients provided informed consent to publish the data. 
Availability of data and material: The datasets used and/or analysed during the current study are available from the corresponding author on reasonable request.

Competing interests: The authors declare that they have no competing interests.

Funding: The study was financially supported by UCB Pharma GmbH. The funding sources had no role in the design and conduct of the study; collection, management, analysis, and interpretation of the data; preparation, review, or approval of the manuscript; or decision to submit the manuscript for publication.

Authors Contribution: $\mathrm{SH}, \mathrm{JK}, \mathrm{QS}, \mathrm{MB}$ and $\mathrm{SO}$ made substantial contributions to the conception or design of the work; or the acquisition, analysis or interpretation of data for the work; SH, JK, QS, AMG, GRB, PH, $\mathrm{GS}, \mathrm{TH}, \mathrm{SH}, \mathrm{MB}, \mathrm{SO}$ were drafting the work or revising it critically for important intellectual content; all authors provide approval for publication of the content and agree to be accountable for all aspects of the work in ensuring that questions related to the accuracy or integrity of any part of the work are appropriately investigated and resolved.

Acknowledgements: We would like to acknowledge all participating patients.

\section{References}

1. Y. Cheng, J. Hootman, L. Murphy, Morbidity and Mortality Weekly Report. Prevalence of doctordiagnosed arthritis and arthritis-attributable activity limitation: United States, 2007-2009. MMWR. Morb. Mortal. Wkly. Rep. (2010).

2. S. A. Fazal, M. Khan, S. E. Nishi, F. Alam, N. Zarin, M. T. Bari, G. M. Ashraf, A Clinical Update and Global Economic Burden of Rheumatoid Arthritis. Endocrine, Metab. Immune Disord. - Drug Targets (2018), doi:10.2174/1871530317666171114122417.

3. S. E. Gabriel, The epidemiology of rheumatoid arthritis. Rheum. Dis. Clin. North Am. (2001), doi:10.1016/S0889-857X(05)70201-5.

4. A. J. Silman, J. E. Pearson, Epidemiology and genetics of rheumatoid arthritis. Arthritis Res. (2002), doi:10.1186/ar578.

5. W. S. Wilke, T. J. Sweeney, L. H. Calabrese, Early, aggressive therapy for rheumatoid arthritis: concerns, descriptions, and estimate of outcome. Semin. Arthritis Rheum. 23, 26-41 (1993).

6. L. E. Burgers, K. Raza, A. H. Van Der Helm-Van Mil, Window of opportunity in rheumatoid arthritisDefinitions and supporting evidence: From old to new perspectives. RMD Open (2019), doi:10.1136/rmdopen-2018-000870.

7. H. Radner, D. Aletaha, Anti-TNF in rheumatoid arthritis: an overview. Wiener Medizinische Wochenschrift (2015), doi:10.1007/s10354-015-0344-y.

8. M. L. L. Prevoo, M. A. Van'T Hof, H. H. Kuper, M. A. Van Leeuwen, L. B. A. Van De Putte, P. L. C. M. Van Riel, Modified disease activity scores that include twenty-eight-joint counts development and 
validation in a prospective longitudinal study of patients with rheumatoid arthritis. Arthritis Rheum. (1995), doi:10.1002/art.1780380107.

9. M. Backhaus, T. Kamradt, D. Sandrock, D. Loreck, J. Fritz, K. J. Wolf, H. Raber, B. Hamm, G. R. Burmester, M. Bollow, Arthritis of the finger joints: A comprehensive approach comparing conventional radiography, scintigraphy, ultrasound, and contrast-enhanced magnetic resonance imaging. Arthritis Rheum. (1999), doi:10.1002/1529-0131(199906)42:6<1232::AID-ANR21>3.0.C0;23.

10. R. J. Wakefield, W. W. Gibbon, P. G. Conaghan, P. O'Connor, D. McGonagle, C. Pease, M. J. Green, D. J. Veale, J. D. Isaacs, P. Emery, The value of sonography in the detection of bone erosions in patients with rheumatoid arthritis: A comparison with conventional radiography. Arthritis Rheum. (2000), doi:10.1002/1529-0131(200012)43:12<2762::aid-anr16>3.0.co;2-\%23.

11. A. N. Colebatch, C. J. Edwards, M. Østergaard, D. Van Der Heijde, P. V. Balint, M. A. D’Agostino, K. Forslind, W. Grassi, E. A. Haavardsholm, G. Haugeberg, A. G. Jurik, R. B. M. Landewé, E. Naredo, P. J. O'Connor, B. Ostendorf, K. Potočki, W. A. Schmidt, J. S. Smolen, S. Sokolovic, I. Watt, P. G. Conaghan, EULAR recommendations for the use of imaging of the joints in the clinical management of rheumatoid arthritis. Ann. Rheum. Dis. (2013), doi:10.1136/annrheumdis-2012-203158.

12. A. Maier, M. Ratanski, M. Renelt, M. Hammer, M. Gehlen, Sonography of the foot in rheumatology: Ultrasound diagnostics of the ankle joint and foot in the rheumatological routine. Z. Rheumatol. (2020), doi:10.1007/s00393-020-00823-1.

13. T. Fischer, I. Gemeinhardt, S. Wagner, D. V. Stieglitz, J. Schnorr, K. G. A. Hermann, B. Ebert, D. Petzelt, R. MacDonald, K. Licha, M. Schirner, V. Krenn, T. Kamradt, M. Taupitz, Assessment of unspecific nearinfrared dyes in laser-induced fluorescence imaging of experimental arthritis. Acad. Radiol. (2006), doi:10.1016/j.acra.2005.07.010.

14. S. Bhasin, P. P. Cheung, The Role of Power Doppler Ultrasonography as Disease Activity Marker in Rheumatoid Arthritis. Dis. Markers (2015),, doi:10.1155/2015/325909.

15. M. Backhaus, S. Ohrndorf, H. Kellner, J. Strunk, T. M. Backhaus, W. Hartung, H. Sattler, K. Albrecht, J. Kaufmann, K. Becker, H. Sörensen, L. Meier, G. R. Burmester, W. A. Schmidt, Evaluation of a novel 7joint ultrasound score in daily rheumatologic practice: A pilot project. Arthritis Care Res. (2009), doi:10.1002/art.24646.

16. T. Fischer, B. Ebert, J. Voigt, R. Macdonald, U. Schneider, A. Thomas, B. Hamm, K. G. A. Hermann, Detection of Rheumatoid Arthritis Using Non-Specific Contrast Enhanced Fluorescence Imaging. Acad. Radiol. (2010), doi:10.1016/j.acra.2009.09.016.

17. A. M. Glimm, S. G. Werner, G. R. Burmester, M. Backhaus, S. Ohrndorf, Analysis of distribution and severity of inflammation in patients with osteoarthitis compared to rheumatoid arthritis by ICGenhanced fluorescence optical imaging and musculoskeletal ultrasound: A pilot study. Ann. Rheum. Dis. 75, 566-570 (2016).

18. M. Krohn, S. Ohrndorf, S. G. Werner, B. Schicke, G. R. Burmester, B. Hamm, M. Backhaus, K. G. A. Hermann, Near-infrared fluorescence optical imaging in early rheumatoid arthritis: A comparison to 
magnetic resonance imaging and ultrasonography. J. Rheumatol. (2015), doi:10.3899/jrheum.141244.

19. R. Meier, C. Krug, D. Golovko, S. Boddington, G. Piontek, M. Rudelius, E. J. Sutton, A. Baur-Melnyk, E. F. Jones, H. E. Daldrup-Link, Indocyanine green - Enhanced imaging of antigen-induced arthritis with an integrated optical imaging/radiography system. Arthritis Rheum. (2010), doi:10.1002/art.27542.

20. S. G. Werner, H. E. Langer, S. Ohrndorf, M. Bahner, P. Schott, C. Schwenke, M. Schirner, H. Bastian, G. Lind-Albrecht, B. Kurtz, G. R. Burmester, M. Backhaus, Inflammation assessment in patients with arthritis using a novel in vivo fluorescence optical imaging technology. Ann. Rheum. Dis. (2012), doi:10.1136/annrheumdis-2010-148288.

21. S. G. Werner, H. E. Langer, P. Schott, M. Bahner, C. Schwenke, G. Lind-Albrecht, F. Spiecker, B. Kurtz, G. R. Burmester, M. Backhaus, Indocyanine green-enhanced fluorescence optical imaging in patients with early and very early arthritis: A comparative study with magnetic resonance imaging. Arthritis Rheum. (2013), doi:10.1002/art.38175.

22. J. Kay, K. S. Upchurch, ACR/EULAR 2010 rheumatoid arthritis classification criteria. Rheumatology. 51, vi5-vi9 (2012).

23. P. ML, van 't H. MA, K. HH, van L. MA, van de P. LB, van R. PL, Modified disease activity scores that include twenty-eight-joint counts. Development and validation in a prospective longitudinal study of patients with rheumatoid arthritis. Arthritis Rheum. 38, 44-48 (1995).

24. J. Fransen, G. Stucki, P. L. C. M. van Riel, Rheumatoid arthritis measures: Disease Activity Score (DAS), Disease Activity Score-28 (DAS28), Rapid Assessment of Disease Activity in Rheumatology (RADAR), and Rheumatoid Arthritis Disease Activity Index (RADAl). Arthritis Care Res. (Hoboken). 49, S214-S224 (2003).

25. A. M. Glimm, L. I. Sprenger, I. K. Haugen, U. Mansmann, S. Hermann, T. Häupl, P. Hoff, G. R. Burmester, M. Backhaus, L. Le, S. Ohrndorf, Fluorescence optical imaging for treatment monitoring in patients with early and active rheumatoid arthritis in a 1-year follow-up period. Arthritis Res. Ther. (2019), doi:10.1186/s13075-019-1989-5.

26. A. K. Scheel, K. G. A. Hermann, S. Ohrndorf, C. Werner, C. Schirmer, J. Detert, M. Bollow, B. Hamm, G. A. Müller, G. R. Burmester, M. Backhaus, Prospective 7 year follow up imaging study comparing radiography, ultrasonography, and magnetic resonance imaging in rheumatoid arthritis finger joints. Ann. Rheum. Dis. (2006), doi:10.1136/ard.2005.041814.

27. M. Szkudlarek, M. Court-Payen, S. Jacobsen, M. Klarlund, H. S. Thomsen, M. Østergaard, Interobserver agreement in ultrasonography of the finger and toe joints in rheumatoid arthritis. Arthritis Rheum. (2003), doi:10.1002/art.10877.

28. J. A. Mendonça, M. A. Yazbek, B. L. Costallat, M. Gutiérrez, M. B. Bértolo, The modified US7 score in the assessment of synovitis in early rheumatoid arthritis. Rev. Bras. Reumatol. (2014), doi:10.1016/j.rbr.2014.03.026.

29. R. Meier, K. Thuermel, P. B. Noël, P. Moog, M. Sievert, C. Ahari, R. A. Nasirudin, D. Golovko, B. Haller, C. Ganter, M. Wildgruber, C. Schaeffeler, S. Waldt, E. J. Rummeny, Synovitis in patients with early 
inflammatory arthritis monitored with quantitative analysis of dynamic contrast-enhanced optical imaging and MR imaging. Radiology. 270, 176-185 (2014).

30. T. Pap, O. Distler, Linking angiogenesis to bone destruction in arthritis. Arthritis Rheum. (2005)," doi:10.1002/art.21015.

31. P. Sarzi-Puttini, E. Filippucci, S. Adami, P. L. Meroni, A. Batticciotto, L. Idolazzi, O. De Lucia, P. Talavera, T. Kumke, W. Grassi, Clinical, Ultrasound, and Predictability Outcomes Following Certolizumab Pegol Treatment (with Methotrexate) in Patients with Moderate-to-Severe Rheumatoid Arthritis: 52-Week Results from the CZP-SPEED Study. Adv. Ther. 35, 1153-1168 (2018).

32. S. L. R. Ali, A.-M. Glimm, G. R. Burmester, P. Hoff, G. Schmittat, S. Hermann, M. Backhaus, J. Klotsche, S. Ohrndorf, Fluorescence optical imaging is helpful in the decision for rituximab (RTX) re-therapy in patients with rheumatoid arthritis (2020), doi:10.21203/rs.3.rs-18466/v1.

33. A. D. do Prado, H. L. Staub, M. C. Bisi, I. G. da Silveira, J. A. Mendonça, J. Polido-Pereira, J. E. Fonseca, Ultrasound and its clinical use in rheumatoid arthritis: where do we stand? Adv. Rheumatol. (London, England) (2018),, doi:10.1186/s42358-018-0023-y.

34. A. A. Razmjou, J. Brook, D. Elashoff, G. Kaeley, S. Choi, T. Kermani, V. K. Ranganath, Ultrasound and multi-biomarker disease activity score for assessing and predicting clinical response to tofacitinib treatment in patients with rheumatoid arthritis. BMC Rheumatol. (2020), doi:10.1186/s41927-02000153-4.

\section{Figures}

\section{Figure 1}

\section{Fluorescence optical imaging illustrations in Phase 1}

A) Baseline visit. FOI findings with clinical active RA. Increased signal intensities as a sign of active inflammation in P1. High signal intensities (FOIAS grade 3) in PIP5 of the right hand, moderate signal intensities (FOIAS grade 2) in both wrists and PIP4 of the right and PIP5 of the left hand.

B) After 52 weeks of CZP treatment; no increased signal intensities are detectable.

\section{Supplementary Files}

This is a list of supplementary files associated with this preprint. Click to download.

- SupplementPaperBIORACHertrampfundOhrndorfFINAL.docx 